\title{
On the Number of Negative Eigenvalues for a Schrödinger Operator with Magnetic Field
}

\section{Zhongwei Shen ${ }^{1}$}

Department of Mathematics, University of Kentucky, Lexington, KY 40506, USA

E-mail: shenz@ms uky edu

Received: 29 February 1996/ Accepted: 14 May 1996

Abstract: We consider the Schrödinger operator with magnetic field

$$
H=\sum_{j=1}^{n}\left(\frac{1}{i} \frac{\partial}{\partial x_{j}}-a_{j}\right)^{2}+V \quad \text { in } \mathbb{R}^{n} .
$$

Under certain conditions on the magnetic field $\mathbf{B}=$ curl $\mathbf{a}$, we generalize the Fefferman-Phong estimates (Bull. A. M. S. 9, 129-206 (1983)) on the number of negative eigenvalues for $-\Delta+V$ to the operator $H$. Upper and lower bounds are established. Our estimates incorporate the contribution from the magnetic field. The conditions on $\mathbf{B}$ in particular are satisfied if the magnetic potentials $a_{j}(x)$ are polynomials.

\section{Introduction}

This paper concerns the Schrödinger operator with magnetic field:

$$
H=H(\mathbf{a}, V)=\sum_{j=1}^{n}\left(\frac{1}{i} \frac{\partial}{\partial x_{j}}-a_{j}\right)^{2}+V \quad \text { in } \mathbb{R}^{n}, \quad n \geqq 3,
$$

where $i=\sqrt{-1}, V: \mathbb{R}^{n} \rightarrow \mathbb{R}$ is the electric potential and $\mathbf{a}: \mathbb{R}^{n} \rightarrow \mathbb{R}^{n}$ is the magnetic potential.

Let $N(\lambda, H)$ denote the number of eigenvalues (counting multiplicity) of $H$ smaller than $\lambda$ (or in general the dimension of the spectral projection for $H$ corresponding to the interval $(-\infty, \lambda))$. In the case $\mathbf{a}(x) \equiv \mathbf{0}$, i.e., $H=H(\mathbf{0}, V)=$ $-\Delta+V$, a basic theorem of Cwickel, Lieb and Rosenblum states that

$$
N(\lambda,-\Delta+V) \leqq c_{n}\left|\left\{(x, \xi) \in \mathbb{R}^{n} \times \mathbb{R}^{n}:|\xi|^{2}+V(x)<\lambda\right\}\right| .
$$

See [Si2, p. 95]. Using a sharper form of the uncertainty principle, C. Fefferman and D.H. Phong were able to refine the classical estimate (0.2). Indeed, it was shown

\footnotetext{
${ }^{1}$ Supported in part by NSF Grant DMS-9596266
} 
in [F] that, for $p>1$ and $\lambda \leqq 0, N(\lambda,-\Delta+V)$ is bounded by $C \cdot N_{0}$, where $N_{0}$ is the number of minimal (disjoint) dyadic cubes which satisfy

$$
l(Q)^{2}\left(\frac{1}{|Q|} \int_{Q}|V|^{p} d x\right)^{1 / p} \geqq c>0, \quad l(Q)<\frac{1}{\sqrt{|\lambda|}},
$$

$l(Q)$ denotes the side length of cube $Q$ and $C, c$ depend only on $n$ and $p$. A lower bound was also established. See [F, p. 145, Theorem 6]. The results of Fefferman and Phong are particularly useful in the cases when the right side of $(0.2)$ becomes infinite [R, Si3, F].

Concerning the case where the magnetic potential $\mathbf{a}(x)$ is present, it is known that estimate $(0.2)$ still holds for $H$ by the diamagnetic inequality, although one can not expect $N(\lambda, H(\mathbf{a}, V)) \leqq N(\lambda, H(\mathbf{0}, V))$. See [A-H-S]. A obvious problem is that this estimate does not involve the magnetic field.

The purpose of this paper is to generalize the Fefferman-Phong estimate to the magnetic Schrödinger operators under certain conditions on the magnetic field $\mathbf{B}=$ curl $\mathbf{a}$. We establish upper and lower bounds of $N(\lambda, H)$ for $\lambda \leqq 0$. The conditions on $\mathbf{B}$ in particular are satisfied if the magnetic potentials $a_{j}(x), j=1,2, \ldots, n$ are polynomials. More importantly, our estimates incorporate the contribution from the magnetic field in an effective way.

To state the main results, we need to introduce an auxiliary function.

Definition 0.4. For a nonnegative function $W$, the function $m(x, W)$ is defined by

$$
\frac{1}{m(x, W)}=\sup \left\{r>0: \frac{r^{2}}{|Q(x, r)|} \int_{Q(x, r)} W(y) d y \leqq 1\right\}
$$

where $Q(x, r)$ denotes the cube centered at $x$ with side length $r$.

The function $m(x,|\mathbf{B}|)$, which behaves like $|\mathbf{B}|^{1 / 2}$ in scale, plays a crucial role in this paper. What makes $m(x,|\mathbf{B}|)$ so important is the fact that, under suitable conditions, we can bound the operator $H(\mathbf{a}, 0)$ from below by $c\{m(x,|\mathbf{B}|)\}^{2}$, i.e.,

$$
(H(\mathbf{a}, 0) f, f) \geqq c(m(\cdot,|\mathbf{B}|) f, m(\cdot,|\mathbf{B}|) f) .
$$

See Theorem 4.1. One may consider the above estimate, which is proved in [Sh3, Theorem 2.7] for a more general case, as a form of the uncertainty principle.

We also need to introduce a class of functions which satisfy the reverse Hölder inequality. This class has been studied extensively in harmonic analysis. See [St2].

Definition 0.6. Suppose $W \in L_{\text {loc }}^{p}\left(\mathbb{R}^{n}\right)(1<p \leqq \infty)$ and $W \geqq 0$ a.e. on $\mathbb{R}^{n}$. We say $W \in(R H)_{p}$ if there exists $C_{0} \geqq 1$ such that

$$
\left(\frac{1}{|Q|} \int_{Q} W^{p}(x) d x\right)^{1 / p} \leqq C_{0} \cdot \frac{1}{|Q|} \int_{Q} W(x) d x
$$

for every cube $Q$ in $\mathbb{R}^{n}$. 
We remark that, if $W=|P(x)|^{\alpha}$, where $P(x)$ is a polynomial of degree $k$ and $\alpha>0$, then $W \in(R H)_{\infty}$ and

$$
m(x, W) \approx \sum_{|\beta| \leqq k}\left|\partial_{x}^{\beta} P(x)\right|^{\frac{\alpha}{\alpha|\beta|+2}}
$$

See [Sh2].

Let

$$
\mathbf{B}(x)=\operatorname{curl} \mathbf{a}(x)=\left(b_{j k}(x)\right)_{1 \leqq j, k \leqq n}
$$

be the magnetic field generated by $\mathbf{a}(x)$, where

$$
b_{j k}(x)=\frac{\partial a_{j}}{\partial x_{k}}-\frac{\partial a_{k}}{\partial x_{j}} .
$$

We are now in a position to state the main results of the paper.

Theorem 0.11. Let $n \geqq 3$. Suppose $\mathbf{a} \in C^{2}\left(\mathbb{R}^{n}\right), V \in L_{\mathrm{loc}}^{p}\left(\mathbb{R}^{n}\right)$ for some $p>1$. Also assume that $|\mathbf{B}| \in(R H)_{n / 2}$ and

$$
|\nabla \mathbf{B}(x)| \leqq C_{1}\{m(x,|\mathbf{B}|)\}^{3},
$$

where $|\mathbf{B}|=|\mathbf{B}(x)|=\sum_{j, k}\left|b_{j k}(x)\right|$. Then, there exist $C=C(n)>0$ and $c=c\left(C_{0}\right.$, $\left.C_{1}, n, p\right)>0$, such that, for $\lambda \leqq 0, N(\lambda, H)$ is bounded by $C \cdot N_{0}$, where $N_{0}$ is the number of minimal (disjoint) dyadic cubes which satisfy

$$
l(Q)^{2}\left(\frac{1}{|Q|} \int_{Q}|V|^{p} d x\right)^{1 / p} \geqq c, \quad l(Q)<\frac{1}{\sqrt{|\lambda|}}
$$

and

$$
l(Q)^{2}\left(\frac{1}{|Q|} \int_{Q}|\mathbf{B}|^{2} d x\right)^{1 / 2} \leqq 1
$$

Remark 0.15 . In Theorem 0.11 , we have implicitly assumed that $H$ has a self-adjoint realization on $L^{2}\left(\mathbb{R}^{n}\right)$. Under the assumption that $\mathbf{a} \in C^{2}\left(\mathbb{R}^{n}\right)$ and $V \in L_{\text {loc }}^{p}\left(\mathbb{R}^{n}\right)$, we may define the quadratic form

$$
q[f, g]=\sum_{j=1}^{n} \int_{\mathbb{R}^{n}}\left(\frac{1}{i} \frac{\partial}{\partial x_{j}}-a_{j}\right) \overline{\left(\frac{1}{i} \frac{\partial}{\partial x_{j}}-a_{j}\right) g} d x+\int_{\mathbb{R}^{n}} V f \bar{g} d x
$$

for $f, g \in C_{0}^{\infty}\left(\mathbb{R}^{n}\right)$. The key estimate (see (0.29) and (0.30) below) of this paper implies that if $M_{p}<\gamma\left(n, p, C_{0}, C_{1}\right)$ for $|\lambda|$ sufficiently large, then $q[f, g]$ is semibounded from below and closable. In this case, $H$ can be extended to the unique self-adjoint operator associated with the quadratic form (0.16).

Remark 0.17. Note that the conditions $|\mathbf{B}| \in(R H)_{n / 2}$ and $|\nabla \mathbf{B}(x)| \leqq C\{m(x,|\mathbf{B}|)\}^{3}$ in Theorem 0.11 are dilation invariant. Roughly speaking, these two conditions mean that the values of $|\mathbf{B}|$ do not fluctuate too much on the average and $|\nabla \mathbf{B}|$ is uniformly bounded in the scale $\{m(x,|\mathbf{B}|)\}^{-1}$. Although some condition on $\nabla \mathbf{B}$ seems to be necessary if $n \geqq 3$, the assumption in Theorem 0.11 is more restrictive than one would hope. Nevertheless, these conditions are satisfied if the magnetic potentials 
$a_{j}(x)$ are polynomials. This follows easily from the estimate (0.8). Moreover, in this case, the constants $C_{0}, C_{1}$ depend only on $n$ and the degrees of polynomials.

Remark 0.18 . In Theorem 0.11 , the condition (0.14) may be replaced by

$$
l(Q)^{2}\left(\frac{1}{|Q|} \int_{Q}|\mathbf{B}|^{q} d x\right)^{1 / q} \leqq 1
$$

for any $0<q \leqq \infty$. Indeed, in the proof of the theorem, we will show that $N(\lambda, H) \leqq C_{n} \cdot \tilde{\hat{N}}_{0}$, where $\tilde{N}_{0}$ is the number of minimal dyadic cubes which satisfy $(0.13)$ and

$$
l(Q)<\inf _{x \in Q} \frac{\alpha}{m(x,|\mathbf{B}|)}
$$

Since (0.12) implies that $|\mathbf{B}(x)| \leqq C\{m(x,|\mathbf{B}|)\}^{2}$ [Sh4, Remark 1.8], we conclude that

$$
l(Q)^{2}\left(\frac{1}{|Q|} \int_{Q}|\mathbf{B}|^{q} d x\right)^{1 / q} \leqq l(Q)^{2} \cdot C \cdot \sup _{x \in Q}\{m(x,|\mathbf{B}|)\}^{2} \leqq \alpha^{2} C \leqq 1
$$

if $\alpha$ is small. then

Note that, if $Q$ is a cube satisfying (0.13) and (0.20) with $\lambda=0$ and $p \geqq n / 2$,

$$
\begin{aligned}
c & \leqq l(Q)^{2-\frac{n}{p}}\left(\int_{Q}|V|^{p} d x\right)^{1 / p} \\
& \leqq\left\{\inf _{x \in Q} \frac{1}{m(x,|\mathbf{B}|)}\right\}^{2-\frac{n}{p}}\left\{\int_{Q}|V|^{p} d x\right\}^{1 / p} \\
& \leqq\left\{\int_{Q} \frac{|V(x)|^{p}}{\{m(x,|\mathbf{B}|)\}^{2 p-n}} d x\right\}^{1 / p} .
\end{aligned}
$$

Thus,

$$
\int_{Q} \frac{|V(x)|^{p}}{\{m(x,|\mathbf{B}|)\}^{2 p-n}} d x \geqq c .
$$

Summing over all minimum cubes which satisfy (0.13) and (0.20), and using Remark 0.18, we obtain the following.

Corollary 0.21. Under the same assumption as in Theorem 0.11 , we have

$$
N(0, H) \leqq C \int_{\mathbb{R}^{n}} \frac{|V(x)|^{p}}{\{m(x,|\mathbf{B}|)\}^{2 p-n}} d x
$$

for $p \geqq n / 2$, where $C$ depends on $n, p, C_{0}$ and $C_{1}$. 
Clearly, we may replace the domain $\mathbb{R}^{n}$ in $(0.22)$ by the set $\left\{x \in \mathbb{R}^{n}: V(x)<0\right\}$. In the case $p=n / 2$, this is the classical Cwickel-Lieb-Rosenblum estimate. We remark that Corollary 0.21 may be deduced directly from estimates $(0.5)$ and the diamagnetic inequality.

Corollary 0.23. Let $\lambda_{j}, j=1, \ldots$, denote the negative eigenvalues of $H$. Under the same assumption as in Theorem 0.11 , we have

$$
\sum_{j}\left|\lambda_{j}\right| \leqq C \int_{\left\{x \in R^{n}: V(x)<0\right\}} \frac{|V(x)|^{p+1}}{\{m(x,|\mathbf{B}|)\}^{2 p-n}} d x
$$

for $p \geqq n / 2$, where $C$ depends on $n, p, C_{0}$, and $C_{1}$.

Corollary $(0.23)$ follows from Corollary $(0.21)$ by a simple integration argument as in the classical case.

Example 0.25. Let $\mathbf{B}(x)$ be a constant magnetic field in $\mathbb{R}^{3}$. Using Corollary $(0.21)$, we get

$$
N(0, H) \leqq \frac{C_{p}}{|\mathbf{B}|^{p-\frac{3}{2}}} \int_{\left\{x \in R^{3}: V(x)<0\right\}}|V|^{p} d x \quad p \geqq 3 / 2 .
$$

The following lower bound estimate suggests that the upper bound in Theorem 0.11 is almost optimal.

Theorem 0.26. Suppose $\mathbf{a} \in C^{1}\left(\mathbb{R}^{n}\right), V \in L_{\mathrm{loc}}^{1}\left(\mathbb{R}^{n}\right)$ and $V \leqq 0$ a.e. on $\mathbb{R}^{n}$. Then, there exists $C_{2}>0$ depending only on $n$, such that, if there exists a collection of cubes $\left\{Q_{k}, k=1,2, \ldots, N_{0}\right\}$, whose doubles are pointwise disjoint, with the properties

$$
l(Q)^{2}\left(\frac{1}{|Q|} \int_{Q}|V| d x\right) \geqq C_{2}, \quad l(Q)<\frac{1}{\sqrt{|\lambda|}}
$$

and

$$
l(Q)^{2}\left(\frac{1}{|Q|} \int_{2 Q}|\mathbf{B}|^{2} d x\right)^{1 / 2} \leqq 1
$$

then

$$
N(\lambda, H) \geqq N_{0} \text {. }
$$

The paper is organized as follows. In Sect. 1 we give the proof of Theorem 0.26 . This will be done by constructing a certain subspace of $L^{2}\left(\mathbb{R}^{n}\right)$ and using the minimax principle. To prove Theorem 0.11 , we follow the approach of Fefferman and Phong [F]. Also see [K-Sa]. The key step, which requires the systematic control over the magnetic field $\mathbf{B}$, is to establish the following trace inequality:

$$
\int_{\mathbb{R}^{n}}|V||g|^{2} d x \leqq C \cdot M_{p}\left\{\sum_{j=1}^{n} \int_{\mathbb{R}^{n}}\left|\left(\frac{1}{i} \frac{\partial}{\partial x_{j}}-a_{j}\right) g\right|^{2} d x+|\lambda| \int_{\mathbb{R}^{n}}|g|^{2} d x\right\},
$$

where

$$
M_{p}=\sup _{Q} l(Q)^{2}\left(\frac{1}{|Q|} \int_{Q}|V|^{p} d x\right)^{1 / p}
$$


and the supremum is over all dyadic cubes satisfying

$$
l(Q)<\inf _{x \in Q} \frac{\alpha}{m(x,|\mathbf{B}|+|\lambda|)} .
$$

Note that $(0.29)$ is equivalent to

$$
\int_{\mathbb{R}^{n}}|V|\left|(H(\mathbf{a}, 0)+|\lambda|)^{-1 / 2} f\right|^{2} d x \leqq C \cdot M_{p} \int_{\mathbb{R}^{n}}|f|^{2} d x .
$$

Let $K_{\lambda}(x, y)$ denote the kernel function of the operator $(H(\mathbf{a}, 0)+|\lambda|)^{-1 / 2}$. In Sect. 2 we will show that, for any integer $k>0$,

$$
\left|K_{\lambda}(x, y)\right| \leqq \frac{C_{k}}{\{1+|x-y| m(x,|\mathbf{B}|+|\lambda|)\}^{k}} \cdot \frac{1}{|x-y|^{n-1}} .
$$

This decay estimate is closely related the lower bound $(0.5)$ for $H(\mathbf{a}, 0)$.

In Sect. 3 we establish the trace inequality $(0.29)$ by using $(0.32)$ and techniques from harmonic analysis. The proof of Theorem 0.11 , which adapts the argument in $[\mathrm{F}, \mathrm{K}-\mathrm{Sa}]$, is given in Sect. 4.

In recent years there has been a great deal of interest in the magnetic Schrödinger operator $H$. For references on the spectral theory of $H$, we refer the reader to a survey paper by Mohamed and Raikov [M-R]. We remark that in [Sh4], under certain conditions similar to that in Theorem 0.11 , we study the eigenvalue asymptotics of $H(\mathbf{a}, V)$ with nonnegative potential $V$. In particular, we show that $H(\mathbf{a}, V)$ has a discrete spectrum if and only if $\lim _{|x| \rightarrow \infty} m(x,|\mathbf{B}|+V)=\infty$.

We fix some notation. By dyadic cubes, we mean cubes in $\mathbb{R}^{n}$ whose side have length $2^{k}$, and whose vertices are members of the lattice of points of the form $\left(m_{1} 2^{k}, \ldots, m_{n} 2^{k}\right)$ with $k, m_{j}$ being arbitrary integers. Throughout this paper, unless otherwise indicated, we will use $C$ and $c$ to denote positive constants, which are not necessarily the same at each occurrence, which depend at most on $C_{0}, C_{1}, n$ and $p$.

Finally, the author would like to thank the referee for several valuable comments.

\section{The Lower Bound}

In this section we will give the proof of Theorem 0.26 stated in the Introduction.

Proof of Theorem 0.26. Suppose that there exists a collection of cubes $\left\{Q_{l}=\right.$ $\left.Q\left(x_{l}, r_{l}\right): l=1,2, \ldots, N_{0}\right\}$ such that, $2 Q_{l_{1}} \cap 2 Q_{l_{2}}=\emptyset$ for $l_{1} \neq l_{2}$,

$$
\frac{r_{l}^{2}}{\left|Q_{l}\right|} \int_{Q_{l}}|V| d x \geqq C_{2}, \quad r_{l}<\frac{1}{\sqrt{|\lambda|}}
$$

and

$$
l\left(Q_{l}\right)^{2}\left(\frac{1}{\left|Q_{l}\right|} \int_{2 Q_{l}}|\mathbf{B}|^{2} d x\right)^{1 / 2} \leqq 1
$$

To prove $N(\lambda, H) \geqq N_{0}$, by the minimax principle, it suffices to show that, there exists a subspace $\mathscr{H}$ such that $\operatorname{dim} \mathscr{H}=N_{0}$ and for any $g \in \mathscr{H}$,

$$
\sum_{j=1}^{n} \int_{\mathbb{R}^{n}}\left|\left(\frac{1}{i} \frac{\partial}{\partial x_{j}}-a_{j}\right) g\right|^{2} d x+\int_{\mathbb{R}^{n}} V|g|^{2} d x \leqq \lambda \int_{\mathbb{R}^{n}}|g|^{2} d x,
$$


i.e.,

$$
\sum_{j=1}^{n} \int_{\mathbb{R}^{n}}\left|\left(\frac{1}{i} \frac{\partial}{\partial x_{j}}-a_{j}\right) g\right|^{2} d x+|\lambda| \int_{\mathbb{R}^{n}}|g|^{2} d x \leqq \int_{\mathbb{R}^{n}}|V||g|^{2} d x,
$$

since $\lambda \leqq 0$ and $V \leqq 0$.

To this end, we let, for $x \in \tilde{Q}_{l}=2 Q_{l}$,

$$
h_{j}^{l}(x)=\frac{1}{\left|\tilde{Q}_{l}\right|} \int_{\tilde{Q}_{l}}\left\{\sum_{k=1}^{n}\left(x_{k}-y_{k}\right) \int_{0}^{1} b_{j k}(y+t(x-y)) t d t\right\} d y
$$

and

$$
\Phi^{l}(x)=\frac{1}{\left|\tilde{Q}_{l}\right|} \int_{\tilde{Q}_{l}}\left\{\sum_{k=1}^{n}\left(x_{k}-y_{k}\right) \int_{0}^{1} a_{k}(y+t(x-y)) d t\right\} d y,
$$

where $x=\left(x_{1}, x_{2}, \ldots, x_{n}\right)$ and $y=\left(y_{1}, y_{2}, \ldots, y_{n}\right)$.

A computation shows that

$$
a_{j}(x)=h_{j}^{l}(x)+\frac{\partial \Phi^{l}}{\partial x_{j}}(x), \quad \text { for } 1 \leqq j \leqq n
$$

and

$$
\int_{\tilde{Q}_{l}}\left|\mathbf{h}^{l}\right|^{2} d x \leqq C \cdot l\left(Q_{l}\right)^{2} \int_{\tilde{Q}_{l}}|\mathbf{B}|^{2} d x
$$

See [I, p. 365].

Note that, by (1.6), for any $g \in C^{1}\left(\mathbb{R}^{n}\right)$,

$$
\left(\frac{1}{i} \frac{\partial}{\partial x_{j}}-a_{j}\right)\left(g e^{i \Phi^{l}}\right)=e^{i \Phi^{l}}\left(\frac{1}{i} \frac{\partial}{\partial x_{j}}-h_{j}^{l}\right) g .
$$

Now, let $\psi_{l} \in C_{0}^{\infty}\left(2 Q_{l}\right)$ such that $\psi \equiv 1$ on $Q_{l}, 0 \leqq \psi_{l} \leqq 1$ and $\left|\nabla \psi_{l}\right| \leqq c_{n} / r_{l}$. Let

$$
\mathscr{H}=\operatorname{Span}\left\{e^{i \Phi^{l}} \psi_{l}, l=1,2, \ldots, N_{0}\right\} .
$$

If $g=e^{i \Phi^{l}} \psi_{l}$, then

$$
\begin{aligned}
& \int_{\mathbb{R}^{n}}\left|\left(\frac{1}{i} \frac{\partial}{\partial x_{j}}-a_{j}\right) g\right|^{2} d x+|\lambda| \int_{\mathbb{R}^{n}}|g|^{2} d x \\
& =\int_{\mathbb{R}^{n}}\left|\left(\frac{1}{i} \frac{\partial}{\partial x_{j}}-h_{j}\right) \psi_{l}\right|^{2} d x+|\lambda| \int_{\mathbb{R}^{n}}\left|\psi_{l}\right|^{2} d x \\
& \leqq 2 \int_{\mathbb{R}^{n}}\left|\nabla \psi_{l}\right|^{2} d x+2 \int_{\mathbb{R}^{n}}\left|\mathbf{h}^{l}\right|^{2}\left|\psi_{l}\right|^{2} d x+|\lambda| \int_{\mathbb{R}^{n}}\left|\psi_{l}\right|^{2} d x \\
& \leqq C\left\{\frac{1}{r_{l}^{2}}\left|Q_{l}\right|+r_{l}^{2} \int_{\tilde{Q}_{l}}|\mathbf{B}|^{2} d x+|\lambda|\left|Q_{l}\right|\right\} \\
& \leqq C \cdot r_{l}^{-2} \cdot\left|Q_{l}\right|,
\end{aligned}
$$

where we have used (1.1) and (1.2) in the last inequality. 
Also note that, by (1.1),

$$
\int_{\mathbb{R}^{n}}|V||g|^{2} d x \geqq \int_{Q_{l}}|V| d x \geqq C_{2} \cdot r_{l}^{-2} \cdot\left|Q_{l}\right|
$$

It follows that (1.3) holds for $g=e^{i \Phi^{l}} \psi_{l}$ if $C_{2}=C_{2}(n)>0$ is sufficiently large. Since $\psi_{l}$ 's have disjoint supports, we conclude that (1.3) holds for any $g \in \mathscr{H}$. This completes the proof of Theorem 0.26 .

\section{Estimates of Kernel Functions}

In this section we will establish a size estimate for the kernel function of the operator $(H(\mathbf{a}, 0)+\lambda)^{-1 / 2}(\lambda>0)$. This estimate will be used in the next section to prove the desired trace inequality.

The following lemma may be found in [Sh1, Lemma 1.4, p. 519].

Lemma 2.1. Suppose $W \in(R H)_{n / 2}$. Then

(a) $m(x, W) \approx m(y, W) \quad$ if $|x-y| \leqq \frac{1}{m(x, W)}$

(b) $m(y, W) \leqq C\{1+|x-y| m(x, W)\}^{k_{0}} m(x, W)$,

(c) $m(x, W) \geqq \frac{c m(y, W)}{\{1+|x-y| m(y, W)\}^{k_{0} /\left(k_{0}+1\right)}}$,

(d) $1+|x-y| m(x, W) \leqq C\{1+|x-y| m(y, W)\}^{k_{0}+1}$

for some $k_{0}>0$.

Since

$$
(H(\mathbf{a}, 0)+\lambda)^{-1 / 2}=\frac{1}{\pi} \int_{0}^{\infty} \alpha^{-1 / 2}(H(\mathbf{a}, 0)+\lambda+\alpha)^{-1} d \alpha,
$$

we shall first estimate the kernel function $\Gamma_{\lambda}(x, y)$ of $(H(\mathbf{a}, 0)+\lambda)^{-1}$.

The following theorem is a special case of Theorem 1.13 in [Sh4].

Theorem 2.3. Let $\mathbf{a} \in C^{2}\left(\mathbb{R}^{n}\right)$ and $\lambda \geqq 1$. Assume that

$$
\left(\frac{1}{|Q|} \int_{Q}|\mathbf{B}|^{n / 2} d x\right)^{2 / n} \leqq C_{0}\left(\lambda+\frac{1}{|Q|} \int_{Q}|\mathbf{B}| d x\right)
$$

for any cube $Q$ with $l(Q) \leqq 1$, and

$$
|\nabla \mathbf{B}(x)| \leqq C_{1}\{m(x,|\mathbf{B}|+\lambda)\}^{3} .
$$

Then

$$
\left|\Gamma_{\lambda}(x, y)\right| \leqq \frac{C_{k}}{\{1+|x-y| m(x,|\mathbf{B}|+\lambda)\}^{k}} \cdot \frac{1}{|x-y|^{n-2}}
$$

for $|x-y| \leqq 1$ and any integer $k>0$. 
Theorem 2.6. Let $\mathbf{a} \in C^{2}\left(\mathbb{R}^{n}\right)$. Assume that $|\mathbf{B}| \in(R H)_{n / 2}$ and

$$
|\nabla \mathbf{B}(x)| \leqq C_{1}\{m(x,|\mathbf{B}|)\}^{3}, \quad x \in \mathbb{R}^{n}
$$

Then

$$
\left|\Gamma_{\lambda}(x, y)\right| \leqq \frac{C_{k}}{\{1+|x-y| m(x,|\mathbf{B}|+\lambda)\}^{k}} \cdot \frac{1}{|x-y|^{n-2}}
$$

for $x, y \in \mathbb{R}^{n}, \lambda>0$ and any integer $k>0$.

Proof. We derive this theorem from Theorem 2.3 by a rescaling argument.

For $0<\varepsilon<\sqrt{\lambda}$, let

$$
a_{j}^{\varepsilon}(x)=\frac{1}{\varepsilon} a_{j}\left(\frac{x}{\varepsilon}\right)
$$

Then

$$
b_{j k}^{\varepsilon}(x)=\frac{\partial a_{j}^{\varepsilon}}{\partial x_{k}}-\frac{\partial a_{k}^{\varepsilon}}{\partial x_{j}}=\frac{1}{\varepsilon^{2}} b_{j k}\left(\frac{x}{\varepsilon}\right) \text {. }
$$

Note that

$$
\left(\frac{1}{i} \frac{\partial}{\partial x_{j}}-a_{j}^{\varepsilon}\right)(g)(x)=\frac{1}{\varepsilon}\left(\frac{1}{i} \frac{\partial}{\partial x_{j}}-a_{j}\right)(f)\left(\frac{x}{\varepsilon}\right),
$$

where $g(x)=f\left(\frac{x}{\varepsilon}\right)$. It follows that

$$
\left(H\left(\mathbf{a}^{\varepsilon}, 0\right)+\frac{\lambda}{\varepsilon^{2}}\right)(g)(x)=\frac{1}{\varepsilon^{2}}(H(\mathbf{a}, 0)+\lambda)(f)\left(\frac{x}{\varepsilon}\right) .
$$

Hence, if $\Gamma_{\lambda}^{\varepsilon}(x, y)$ denotes the kernel function for $\left(H\left(\mathbf{a}^{\varepsilon}, 0\right)+\frac{\lambda}{\varepsilon^{2}}\right)^{-1}$, we have

$$
\Gamma_{\lambda}(x, y)=\varepsilon^{n-2} \Gamma_{\lambda}^{\varepsilon}(\varepsilon x, \varepsilon y) .
$$

Finally, note that, $\left|\mathbf{B}^{\varepsilon}\right| \in(R H)_{n / 2}$ with a constant $C_{0}$ independent of $\varepsilon>0$,

$$
m\left(x,\left|\mathbf{B}^{\varepsilon}\right|+\frac{\lambda}{\varepsilon^{2}}\right)=\frac{1}{\varepsilon} m\left(\frac{x}{\varepsilon},|\mathbf{B}|+\lambda\right),
$$

where $\mathbf{B}^{\varepsilon}=\left(b_{j k}^{\varepsilon}\right)$, and

$$
\begin{aligned}
\left|\nabla \mathbf{B}^{\varepsilon}(x)\right| & =\frac{1}{\varepsilon^{3}}\left|\nabla \mathbf{B}\left(\frac{x}{\varepsilon}\right)\right| \leqq \frac{C_{1}}{\varepsilon^{3}}\left\{m\left(\frac{x}{\varepsilon},|\mathbf{B}|\right)\right\}^{3} \leqq \frac{C_{1}}{\varepsilon^{3}}\left\{m\left(\frac{x}{\varepsilon},|\mathbf{B}|+\lambda\right)\right\}^{3} \\
& =C_{1}\left\{m\left(x,\left|\mathbf{B}^{\varepsilon}\right|+\frac{\lambda}{\varepsilon^{2}}\right)\right\}^{3}
\end{aligned}
$$

by (2.7) and (2.13). Thus, by (2.12) and Theorem 2.3, if $|x-y| \leqq \frac{1}{\varepsilon}$,

$$
\begin{aligned}
\left|\Gamma_{\lambda}(x, y)\right| & \leqq \frac{C_{k} \cdot \varepsilon^{n-2}}{\left\{1+|\varepsilon x-\varepsilon y| m\left(\varepsilon x,\left|\mathbf{B}^{\varepsilon}\right|+\frac{\lambda}{\varepsilon^{2}}\right)\right\}^{k}} \cdot \frac{1}{|\varepsilon x-\varepsilon y|^{n-2}} \\
& =\frac{C_{k}}{\left\{1+\varepsilon|x-y| m\left(\varepsilon x,\left|\mathbf{B}^{\varepsilon}\right|+\frac{\lambda}{\varepsilon^{2}}\right)\right\}^{k}} \cdot \frac{1}{|x-y|^{n-2}} \\
& =\frac{C_{k}}{\{1+|x-y| m(x,|\mathbf{B}|+\lambda)\}^{k}} \cdot \frac{1}{|x-y|^{n-2}},
\end{aligned}
$$

where we have used (2.13) in the last step. 
The proof is then finished since $\varepsilon$ can be made arbitrarily small.

Using (2.2), we may write

$$
(H(\mathbf{a}, 0)+\lambda)^{-1 / 2} f(x)=\int_{\mathbb{R}^{n}} K_{\lambda}(x, y) f(y) d y,
$$

where

$$
K_{\lambda}(x, y)=\frac{1}{\pi} \int_{0}^{\infty} \alpha^{-1 / 2} \Gamma_{\lambda+\alpha}(x, y) d \alpha .
$$

Theorem 2.16. Under the same hypothesis as in Theorem 2.6, we have

$$
\left|K_{\lambda}(x, y)\right| \leqq \frac{C_{k}}{\{1+|x-y| m(x,|\mathbf{B}|+\lambda)\}^{k}} \cdot \frac{1}{|x-y|^{n-1}}
$$

for $x, y \in \mathbb{R}^{n}, \lambda>0$ and any integer $k>0$.

Proof. It follows from (2.15) and Theorem 2.6 that

$$
\begin{aligned}
\left|K_{\lambda}(x, y)\right| & \leqq \frac{C_{k}}{|x-y|^{n-2}} \int_{0}^{\infty} \frac{\alpha^{-1 / 2} d \alpha}{\{1+|x-y| m(x,|\mathbf{B}|+\lambda+\alpha)\}^{k}} \\
& \leqq \frac{1}{|x-y|^{n-2}} \cdot \frac{C_{k}}{\{1+|x-y| m(x,|\mathbf{B}|+\lambda)\}^{k-2}} \int_{0}^{\infty} \frac{\alpha^{-1 / 2} d \alpha}{\{1+|x-y| \sqrt{\alpha}\}^{2}} \\
& \leqq \frac{C_{k}}{\{1+|x-y| m(x,|\mathbf{B}|+\lambda)\}^{k-2}} \cdot \frac{1}{|x-y|^{n-1}},
\end{aligned}
$$

where in the second inequality we have used $m(x,|\mathbf{B}|+\lambda+\alpha) \geqq m(x,|\mathbf{B}|+\lambda)$ and $m(x,|\mathbf{B}|+\lambda+\alpha) \geqq \sqrt{\alpha}$.

The theorem then follows since $k>0$ is arbitrary.

Remark 2.17. By part (d) of Lemma 2.1, we also have

$$
\left|K_{\lambda}(x, y)\right| \leqq \frac{C_{k}}{\{1+|x-y| m(y,|\mathbf{B}|+\lambda)\}^{k}} \cdot \frac{1}{|x-y|^{n-1}} .
$$

\section{A Trace Inequality}

This section is devoted to the proof of the following trace inequality.

Theorem 3.1. Suppose that $\mathbf{a} \in C^{2}\left(\mathbb{R}^{n}\right)$ and $V \in L_{\mathrm{loc}}^{p}\left(\mathbb{R}^{n}\right)$ for some $p>1$ Also assume that $|\mathbf{B}| \in(R H)_{n / 2}$ and

$$
|\nabla \mathbf{B}(x)| \leqq C_{2}\{m(x,|\mathbf{B}|)\}^{3} \quad \text { on } \mathbb{R}^{n} .
$$

Then, for $g \in C_{0}^{1}\left(\mathbb{R}^{n}\right)$ and $\lambda \geqq 0$,

$$
\int_{\mathbb{R}^{n}}|V||g|^{2} d x \leqq C \cdot M_{p}\left\{\sum_{j=1}^{n} \int_{\mathbb{R}^{n}}\left|\left(\frac{1}{i} \frac{\partial}{\partial x_{j}}-a_{j}\right) g\right|^{2} d x+\lambda \int_{\mathbb{R}^{n}}|g|^{2} d x\right\}
$$


where

$$
M_{p}=\sup _{Q} l(Q)^{2}\left(\frac{1}{|Q|} \int_{Q}|V|^{p} d x\right)^{1 / p}
$$

and the supremum is over all dyadic cubes $Q$ with the property

$$
l(Q)<\inf _{x \in Q} \frac{\alpha}{m(x,|\mathbf{B}|)+\sqrt{\lambda}}
$$

and $C$ depends on $C_{0}, C_{1}, n, p$ and $\alpha$ (to be chosen later).

It is not hard to see that (3.2) is equivalent to

$$
\int_{\mathbb{R}^{n}}|V|\left|(H+\lambda)^{-1 / 2} f\right|^{2} d x \leqq C \cdot M_{p} \int_{\mathbb{R}^{n}}|f|^{2} d x .
$$

To show (3.3), we note that, by (2.18),

$$
\begin{aligned}
\left|(H+\lambda)^{-1 / 2} f(x)\right| \leqq & C \int_{R^{n}} \frac{|f(y)| d y}{\{1+m(y,|\mathbf{B}|+\lambda)|x-y|\}^{k}|x-y|^{n-1}} \\
\leqq & C \int_{|x-y|<\frac{c_{0}}{m(y,|\mathbf{B}|+\lambda)}} \frac{|f(y)| d y}{|x-y|^{n-1}} \\
& +C \quad \int_{|x-y| \geqq \frac{c_{0}}{m(y,|\mathbf{B}|+\lambda)}} \frac{|f(y)| d y}{\{m(y,|\mathbf{B}|+\lambda)|x-y|\}^{k}|x-y|^{n-1}} \\
= & C\left\{T_{1}(|f|)(x)+T_{2}(|f|)(x)\right\},
\end{aligned}
$$

where $c_{0}>0$ is a small constant to be determined later.

The desired estimate of $T_{2}(|f|)$ is fairly straightforward.

Lemma 3.4. Under the same assumption as in Theorem 3.1, we have

$$
\int_{\mathbb{R}^{n}}|V|\left|T_{2} f\right|^{2} d x \leqq C \cdot M_{1} \int_{\mathbb{R}^{n}}|f|^{2} d x
$$

Proof. Note that, by part (d) of Lemma 2.1,

$$
\int_{|x-y| \geqq \frac{c_{0}}{m(y,|\mathbf{B}|+\lambda)}} \frac{d y}{\{m(y,|\mathbf{B}|+\lambda)|x-y|\}^{k}|x-y|^{n-1}} \leqq \frac{C}{m(x,|\mathbf{B}|+\lambda)} .
$$

Thus, by the Cauchy inequality, $T_{2} f(x)$ is bounded by

$$
\frac{C}{\{m(x,|\mathbf{B}|+\lambda)\}^{1 / 2}}\left\{\int_{|x-y| \geqq \frac{c_{0}}{m(y,|\mathbf{B}|+\lambda)}} \frac{|f(y)|^{2} d y}{\{m(y,|\mathbf{B}|+\lambda)|x-y|\}^{k}|x-y|^{n-1}}\right\}^{1 / 2} .
$$


It follows that

$$
\begin{aligned}
& \int_{\mathbb{R}^{n}}|V(x)|\left|T_{2} f(x)\right|^{2} d x \leqq C \int_{\mathbb{R}^{n}}|f(y)|^{2} d y \\
& \quad \times\left\{\begin{array}{c}
\int c_{0} \\
|x-y| \geqq \frac{c_{0}}{m(y,|\mathbf{B}|+\lambda)}
\end{array}\right. \\
& \left.\quad \frac{|V(x)| d x}{m(x,|\mathbf{B}|+\lambda)\{m(y,|\mathbf{B}|+\lambda)|x-y|\}^{k}|x-y|^{n-1}}\right\} .
\end{aligned}
$$

We will show that

$$
\int_{|x-y| \geqq \frac{c_{0}}{m(y,|\mathbf{B}|+\lambda)}} \frac{|V(x)| d x}{m(x,|\mathbf{B}|+\lambda)\{m(y,|\mathbf{B}|+\lambda)|x-y|\}^{k}|x-y|^{n-1}} \leqq C \cdot M_{1} .
$$

Clearly, this gives the estimate in the lemma.

To see (3.5), note that, if $t$ is an integer and $2^{t-1}>c_{0}$,

$$
\begin{gathered}
\int_{|x-y| \approx \frac{2^{t}}{m(y,|\mathbf{B}|+\lambda)}} \frac{|V(x)| d x}{m(x,|\mathbf{B}|+\lambda)\{m(y,|\mathbf{B}|+\lambda)|x-y|\}^{k}|x-y|^{n-1}} \\
\leqq \frac{C\{m(y,|\mathbf{B}|+\lambda)\}^{n-1}}{\left(2^{t}\right)^{k+n-1}} \int_{|x-y| \approx \frac{2^{t}}{m(y,|\mathbf{B}|+\lambda)}} \frac{|V(x)| d x}{m(x,|\mathbf{B}|+\lambda)} \\
\leqq \frac{C\{m(y,|\mathbf{B}|+\lambda)\}^{n-2}}{\left(2^{t}\right)^{k+n-1-\frac{k_{0}}{k_{0}+1}}} \int_{E(y)}|V(x)| d x,
\end{gathered}
$$

where $E(y)$ is the ball centered at $y$ with radius $C 2^{t} / m(y,|\mathbf{B}|+\lambda)$ and we have used part (c) of Lemma 2.1 in the last inequality.

Now, fix $y$, we may cover $E(y)$ by a collection of cubes $\left\{Q_{l}=Q\left(x_{l}, r_{l}\right)\right\}$ with $r_{l}=1 / m\left(x_{l},|\mathbf{B}|+\lambda\right)$ such that $x_{l} \in E(y)$ and $\left\{Q\left(x_{l}, r_{l} / 5\right), l=1,2, \ldots\right\}$ are disjoint. Then

$$
\begin{aligned}
& \int_{E(y)}|V(x)| d x \leqq \sum_{l} \int_{Q_{l}}|V(x)| d x \leqq M_{1} \sum_{l} r_{l}^{n-2} \\
& =M_{1} \sum_{l} r_{l}^{n} \cdot\left\{m\left(x_{l},|\mathbf{B}|+\lambda\right)\right\}^{2} \\
& \leqq C \cdot M_{1} \cdot\left(2^{t}\right)^{2 k_{0}} \cdot\{m(y,|\mathbf{B}|+\lambda)\}^{2} \sum_{l}\left|Q\left(x_{l}, \frac{r_{l}}{5}\right)\right| \\
& \leqq C \cdot M_{1} \cdot\left(2^{t}\right)^{2 k_{0}} \cdot\{m(y,|\mathbf{B}|+\lambda)\}^{2}\left|\left\{x \in \mathbb{R}^{n}:|x-y| \leqq \frac{C 2^{t}}{m(y,|\mathbf{B}|+\lambda)}\right\}\right| \\
& \leqq C \cdot M_{1} \cdot\left(2^{t}\right)^{2 k_{0}+n}\{m(y,|\mathbf{B}|+\lambda)\}^{2-n},
\end{aligned}
$$


where we have used the fact that, for $x \in Q\left(x_{l}, r_{l} / 5\right)$,

$$
\begin{aligned}
|x-y| & \leqq\left|x-x_{l}\right|+\left|x_{l}-y\right| \leqq \frac{C\left\{2^{t}+\left(1+2^{t}\right)^{k_{0} /\left(k_{0}+1\right)}\right\}}{m(y,|\mathbf{B}|+\lambda)} \\
& \leqq \frac{C 2^{t}}{m(y,|\mathbf{B}|+\lambda)} .
\end{aligned}
$$

Thus,

$$
\int_{|x-y| \approx \frac{2^{t}}{m(y,|\mathbf{B}|+\lambda)}} \frac{|V(x)| d x}{m(x,|\mathbf{B}|+\lambda)\{m(y,|\mathbf{B}|+\lambda)|x-y|\}^{k}|x-y|^{n-1}} \leqq \frac{C \cdot M_{1}}{\left(2^{t}\right)^{k-\frac{k_{0}}{k_{0}+1}-2 k_{0}-1}} .
$$

Equation (3.5) then follows easily if we choose $k$ sufficiently large and sum up the above estimate over $t$. This completes the proof of Lemma 3.4.

It remains to show that

$$
\int_{\mathbb{R}^{n}}\left|T_{1} f\right|^{2}|V| d x \leqq C \cdot M_{p} \int_{\mathbb{R}^{n}}|f|^{2} d x,
$$

where

$$
T_{1} f(x)=\int_{|x-y|<\frac{c_{0}}{m(y,|\mathbf{B}|+\lambda)}} \frac{f(y) d y}{|x-y|^{n-1}} .
$$

Our approach to $T_{1} f$ will be similar to that in the case of $-\Delta+V$, where the corresponding operator is

$$
(-\Delta)^{-1 / 2} f(x)=c_{n} \int_{\mathbb{R}^{n}} \frac{f(y) d y}{|x-y|^{n-1}} .
$$

See $[F, \mathrm{~K}-\mathrm{Sa}]$.

Let

$$
V^{+}(x)=\sup _{Q \ni x}\left(\frac{1}{|Q|} \int_{Q}|V|^{p} d x\right)^{1 / p},
$$

where the supremum is over all dyadic cubes such that $x \in Q$ and

$$
l(Q)<\inf _{y \in Q} \frac{\alpha}{m(y,|\mathbf{B}|)+\sqrt{\lambda}} .
$$

Clearly, $|V(x)| \leqq V^{+}(x)$. We will show that

$$
\int_{\mathbb{R}^{n}}\left|T_{1} f\right|^{2} V^{+} d x \leqq C \cdot M_{p} \int_{\mathbb{R}^{n}}|f|^{2} d x
$$

Note that

$$
\int_{\mathbb{R}^{n}} T_{1} f(x) g(x) V^{+}(x) d x=\int_{\mathbb{R}^{n}} f(y)\left\{\int_{|x-y|<\frac{c_{0}}{m(y,|\mathbf{B}|+\lambda)}} \frac{g(x) V^{+}(x) d x}{|x-y|^{n-1}}\right\} d y .
$$

By duality, it suffices to show that

$$
\int_{\mathbb{R}^{n}}\left|T\left(g V^{+}\right)\right|^{2} d x \leqq C \cdot M_{p} \int_{\mathbb{R}^{n}}|g|^{2} V^{+} d x,
$$


where

$$
T g(x)=\int_{|y-x|<\frac{c_{0}}{m(x,|\mathbf{B}|+\lambda)}} \frac{g(y) d y}{|x-y|^{n-1}}
$$

Lemma 3.13. Let

$$
M f(x)=\sup _{Q \ni x} \frac{1}{l(Q)^{n-1}} \int_{Q}|f(y)| d y,
$$

where the supremum is over all cubes $Q$ such that $x \in Q$ and

$$
l(Q)<\inf _{y \in Q} \frac{C_{4} c_{0}}{m(y,|\mathbf{B}|+\lambda)}
$$

Then, for $0<q<\infty$,

$$
\int_{\mathbb{R}^{n}}|T f|^{q} d x \leqq C_{q} \int_{\mathbb{R}^{n}}|M f|^{q} d x
$$

Proof. Without loss of generality, we may assume that $f \geqq 0$ a.e.

Let $\beta>0$ and

$$
E=\left\{x \in \mathbb{R}^{n}:|T f(x)|>\beta\right\}
$$

By the Whitney decomposition [St1, p. 167], there exists a collection of disjoint dyadic cubes $\left\{Q_{l}, l=1,2, \ldots\right\}$ such that $E=\bigcup_{l} Q_{l}$ and $5 Q_{l} \cap\left(\mathbb{R}^{n} \backslash E\right) \neq \emptyset$.

We will show that, for $\gamma>0$ small,

$$
\left|\left\{x \in Q_{l}:|T f(x)|>2 \beta, M f(x) \leqq \gamma \beta\right\}\right| \leqq C \gamma^{\frac{n}{n-1}}\left|Q_{l}\right|
$$

This implies the estimate in the lemma. Indeed, summing over all cubes $Q_{l}$, we obtain

$$
\begin{aligned}
& \left|\left\{x \in \mathbb{R}^{n}:|T f(x)|>2 \beta\right\}\right| \leqq\left|\left\{x \in \mathbb{R}^{n}: M f(x)>\gamma \beta\right\}\right| \\
& \quad+C \gamma^{\frac{n}{n-1}}\left|\left\{x \in \mathbb{R}^{n}:|T f(x)|>\beta\right\}\right| .
\end{aligned}
$$

We then multiply both sides of this inequality by $q \beta^{q-1}$ and integrate in $\beta$ over $(0, \infty)$. The estimate in the lemma follows by choosing $\gamma$ small and bringing the second integral in the right side to the left.

To show (3.14), we fix $Q=Q_{l}=Q\left(x_{0}, r_{0}\right)$. We need to consider two cases.

We begin with the case when

$$
r_{0}<\frac{\varepsilon c_{0}}{m\left(x_{0},|\mathbf{B}|+\lambda\right)}
$$


Let $f=f_{1}+f_{2}$, where $f_{1}=f \chi_{6 Q}$. Let $z \in 5 Q \cap\left(\mathbb{R}^{n} \backslash E\right)$. For any $x \in Q$, we write

$$
\begin{aligned}
& T\left(f_{2}\right)(x)-T\left(f_{2}\right)(z) \\
& =\int_{\substack{|y-x|<\frac{c_{0}}{m(x,|\mathbf{B}|+\lambda)} \\
|y-z| \geqq \frac{c_{0}}{m(z,|\mathbf{B}|+\lambda)}}} \frac{f_{2}(y) d y}{|y-x|^{n-1}}-\int_{\substack{|y-z|<\frac{c_{0}}{m(z,|\mathbf{B}|+\lambda)} \\
|y-x| \geqq \frac{c_{0}}{m(x,|\mathbf{B}|+\lambda)}}} \frac{f_{2}(y) d y}{|y-z|^{n-1}} \\
& +\int_{\substack{|y-x|<\frac{c_{0}}{m(x,|\mathbf{B}|+\lambda)} \\
|y-z|<\frac{c_{0}}{m(z,|\mathbf{B}|+\lambda)}}} f_{2}(y)\left\{\frac{1}{|y-x|^{n-1}}-\frac{1}{|y-z|^{n-1}}\right\} d y \\
& =I_{1}+I_{2}+I_{3} .
\end{aligned}
$$

Note that, by (3.15) and part (a) of Lemma 2.1, $m(w,|\mathbf{B}|+\lambda) \approx m\left(x_{0},|\mathbf{B}|+\lambda\right)$ if $w \in 5 Q$. Thus, for $I_{1}$,

$$
\begin{aligned}
|y-x| & \geqq|y-z|-|z-x| \geqq \frac{c_{0}}{m(z,|\mathbf{B}|+\lambda)}-c_{n} l(Q) \\
& \geqq \frac{c_{0}}{m(z,|\mathbf{B}|+\lambda)}-\frac{\varepsilon c_{n} c_{0}}{m\left(x_{0},|\mathbf{B}|+\lambda\right)} \geqq \frac{c}{m(x,|\mathbf{B}|+\lambda)}
\end{aligned}
$$

if $\varepsilon$ is small.

It follows that

$$
\begin{aligned}
\left|I_{1}\right| & \leqq C \cdot\{m(x,|\mathbf{B}|+\lambda)\}^{n-1} \int_{|y-x|<\frac{c_{0}}{m(x,|\mathbf{B}|+\lambda)}} f(y) d y \\
& \leqq C M f(x) .
\end{aligned}
$$

Similarly,

$$
\begin{aligned}
\left|I_{2}\right| & \leqq C \cdot\{m(z,|\mathbf{B}|+\lambda)\}^{n-1} \int_{|y-z|<\frac{c_{0}}{m(z,|\mathbf{B}|+\lambda)}} f(y) d y \\
& \leqq C \cdot\{m(x,|\mathbf{B}|+\lambda)\}^{n-1} \int_{|y-x|<\frac{C c_{0}}{m(x,|\mathbf{B}|+\lambda)}} f(y) d y \\
& \leqq C M f(x) .
\end{aligned}
$$

To estimate $I_{3}$, note that

$$
\begin{aligned}
\left|I_{3}\right| & \leqq C \int_{|y-x|<\frac{c_{0}}{m(x,|\mathbf{B}|+\lambda)}} f_{2}(y) \cdot \frac{|x-z|}{|y-x|^{n}} d y \\
& \leqq C \sum_{j=0}^{\infty} \frac{1}{\left[2^{j} l(Q)\right]^{n-1}} \int_{\substack{|y-x| \sim 2^{j} l(Q) \\
|y-x|<\frac{c_{0}}{m(x, \mathbf{B} \mid+\lambda)}}} f(y) d y \cdot \frac{1}{2^{\jmath}} \\
& \leqq C M f(x) .
\end{aligned}
$$


This, together with estimates for $I_{1}$ and $I_{2}$, shows that

$$
\begin{aligned}
\left|T f_{2}(x)\right| & \leqq\left|T\left(f_{2}\right)(z)\right|+C M f(x) \leqq|T(f)(z)|+C M f(x) \\
& \leqq \beta+C \gamma \beta \leqq 3 \beta / 2
\end{aligned}
$$

if $M f(x) \leqq \gamma \beta$, and $\gamma$ is small.

Thus,

$$
\begin{aligned}
\mid\{x & \in Q:|T f(x)|>2 \beta, M f(x) \leqq \gamma \beta\} \mid \\
& \leqq\left|\left\{x \in Q:\left|T\left(f_{1}\right)(x)\right|>\beta / 2, M f(x) \leqq \gamma \beta\right\}\right| \\
& \leqq\left|\left\{x \in \mathbb{R}^{n}:\left|(-\Delta)^{-1 / 2}\left(f_{1}\right)(x)\right|>c_{n} \beta\right\}\right| \\
& \leqq\left(\frac{C_{n}}{\beta} \int_{\mathbb{R}^{n}}\left|f_{1}\right| d x\right)^{\frac{n}{n-1}} \leqq \frac{C_{n}}{\beta^{\frac{n}{n-1}}}\left(\int_{6 Q}|f| d x\right)^{\frac{n}{n-1}} \\
& \leqq \frac{C_{n}}{\beta^{\frac{n}{n-1}}} \cdot|Q| \cdot\left(\frac{1}{l(6 Q)^{n-1}} \int_{6 Q}|f| d x\right)^{\frac{n}{n-1}} \\
& \leqq \frac{C_{n}}{\beta^{\frac{n}{n-1}}} \cdot|Q| \cdot(\gamma \beta)^{\frac{n}{n-1}}=C_{n} \gamma^{\frac{n}{n-1}}|Q|
\end{aligned}
$$

where we have used the weak-type $\left(1, \frac{n}{n-1}\right)$ estimate for the fractional integral $(-\Delta)^{-1 / 2}$ (see [St1, p. 119-120]) in the third inequality.

Next, we consider the case when

$$
r_{0} \geqq \frac{\varepsilon c_{0}}{m\left(x_{0},|\mathbf{B}|+\lambda\right)} .
$$

In this case, we bisect $Q$ repeatedly, stopping at $I_{k}=Q\left(x_{k}, r_{k}\right)$ if

$$
r_{k}<\frac{\varepsilon c_{0}}{m\left(x_{k},|\mathbf{B}|+\lambda\right)}
$$

We then obtain $Q=\bigcup_{k} I_{k}$. Since $I_{k}$ is a maximal element among subcubes which satisfy (3.16), by part (a) of Lemma 2.1,

$$
r_{k} \geqq \frac{c_{0}}{C m\left(x_{k},|\mathbf{B}|+\lambda\right)} .
$$

Note that, if $x \in I_{k}$ and $|y-x|<c_{0} / m(x,|\mathbf{B}|+\lambda)$,

$$
\left|y-x_{k}\right| \leqq|y-x|+\left|x-x_{k}\right| \leqq \frac{c_{0}}{m(x,|\mathbf{B}|+\lambda)}+\sqrt{n} r_{k} \leqq \frac{C c_{0}}{m\left(x_{k},|\mathbf{B}|+\lambda\right)} \text {. }
$$

Now let $\widetilde{I_{k}}=Q\left(x_{k}, C c_{0} / m\left(x_{k},|\mathbf{B}|+\lambda\right)\right)$. By (3.16), (3.17) and (3.18), $\left|I_{k}\right| \approx\left|\widetilde{I_{k}}\right|$,

$$
\begin{gathered}
l\left(\widetilde{I_{k}}\right)=\frac{C c_{0}}{m\left(x_{k},|\mathbf{B}|+\lambda\right)} \leqq \inf _{y \in \widetilde{I}_{k}} \frac{C_{4} c_{0}}{m(y,|\mathbf{B}|+\lambda)}, \\
T f(x)=T\left(f \chi_{\widetilde{I}_{k}}\right)(x) \text { for } x \in I_{k} .
\end{gathered}
$$


It then follows from the weak type $\left(1, \frac{n}{n-1}\right)$ estimates of the fractional integral $(-\Delta)^{-1 / 2}[$ St1, p. 119] that

$$
\begin{aligned}
\mid\{x & \in Q:|T(f)(x)|>2 \beta, M f(x) \leqq \gamma \beta\} \mid \\
& \leqq \sum_{k}\left|\left\{x \in I_{k}:(-\Delta)^{-1 / 2}\left(f \chi_{\widetilde{I_{k}}}\right)(x)>c \beta, M f(x) \leqq \gamma \beta\right\}\right| \\
& \leqq \frac{C}{\beta^{\frac{n}{n-1}}} \sum_{k}\left(\int_{\widetilde{I_{k}}}|f(x)| d x\right)^{n /(n-1)} \\
& \leqq \frac{C}{\beta^{\frac{n}{n-1}}} \sum_{k}\left|\widetilde{I_{k}}\right| \cdot(\gamma \beta)^{n /(n-1)} \leqq C \gamma^{\frac{n}{n-1}}|Q|,
\end{aligned}
$$

where we have assumed that $\left\{x \in I_{k}: M f(x) \leqq \gamma \beta\right\} \neq \emptyset$.

The proof of Lemma 3.13 is now complete.

Let

$$
M^{d y} f(x)=\sup _{Q \ni x} \frac{1}{l(Q)^{n-1}} \int_{Q}|f(y)| d y,
$$

where the supremum is taken over all dyadic cubes with the property

$$
l(Q)<\inf _{y \in Q} \frac{C_{4}^{2} c_{0}}{m(y,|\mathbf{B}|+\lambda)} .
$$

Lemma 3.21. For $0<q<\infty$, we have

$$
\int_{\mathbb{R}^{n}}|M f(x)|^{q} d x \leqq C_{q} \int_{\mathbb{R}^{n}}\left|M^{d y} f(x)\right|^{q} d x
$$

Proof. The lemma follows from

$$
\left|\left\{x \in \mathbb{R}^{n}: M f(x)>\beta\right\}\right| \leqq c_{n}\left|\left\{x \in \mathbb{R}^{n}: M^{d y} f(x)>2^{-n} \beta\right\}\right| .
$$

We omit the details. See [K-Sa, p. 215] for a similar estimate.

Finally, we have to show

$$
\int_{\mathbb{R}^{n}}\left|M^{d y}\left(f V^{+}\right)\right|^{2} d x \leqq C \cdot M_{p} \int_{\mathbb{R}^{n}}|f|^{2} V^{+} d x
$$

Lemma 3.24. Suppose that $Q$ is a dyadic cube which satisfies (3.9). Then

$$
\frac{1}{|Q|} \int_{Q} V^{+}(x) d x \leqq C \sup _{Q^{\prime} \supseteq Q}\left(\frac{1}{\left|Q^{\prime}\right|} \int_{Q^{\prime}}|V(x)|^{p} d x\right)^{1 / p},
$$

where the supremum is over all dyadic cubes $Q^{\prime}$ which contain $Q$ and satisfy (3.9). 
Proof. Let $c(Q)$ denote the supremum in the right side of (3.25) and

$$
V_{Q}^{+}(x)=\sup _{\substack{Q^{\prime} \subset Q, x \in Q^{\prime} \\ Q^{\prime} \text { dyadic }}}\left(\frac{1}{\left|Q^{\prime}\right|} \int_{Q^{\prime}}|V(y)|^{p} d y\right)^{1 / p}
$$

Then, for $x \in Q$,

$$
V^{+}(x) \leqq c(Q)+V_{Q}^{+}(x)
$$

By the maximal theorem,

$$
\begin{aligned}
\frac{1}{|Q|} \int_{Q} V^{+} d x & \leqq c(Q)+\frac{1}{|Q|} \int_{Q} V_{Q}^{+}(x) d x \\
& \leqq c(Q)+C\left(\frac{1}{|Q|} \int_{Q}|V|^{p} d x\right)^{1 / p} \\
& \leqq C \cdot c(Q) .
\end{aligned}
$$

Lemma 3.24 is proved.

It follows from (3.25) that

$$
\frac{1}{|Q|} \int_{Q} V^{+}(x) d x \leqq C \cdot \inf _{x \in Q} V^{+}(x),
$$

i.e., $V^{+}(x)$ is an $A_{1}$ weight on cubes satisfying (3.9). Thus, if $E \subset Q$ and $Q$ satisfies (3.9),

$$
\int_{E} V^{+}(x) d x \geqq|E| \cdot \inf _{x \in E} V^{+}(x) \geqq|E| \cdot \inf _{x \in Q} V^{+}(x) \geqq \frac{|E|}{C} \cdot \frac{1}{|Q|} \int_{Q} V^{+}(x) d x .
$$

Hence,

$$
\frac{\int_{E} V^{+} d x}{\int_{Q} V^{+} d x} \geqq \frac{1}{C} \cdot \frac{|E|}{|Q|}
$$

Let

$$
L=\sup _{Q} \frac{1}{l(Q)^{n-2}} \int_{Q} V^{+}(x) d x
$$

where supremum is over all dyadic cubes with the property

$$
l(Q)<\inf _{x \in Q} \frac{C_{4}^{2} c_{0}}{m(x,|\mathbf{B}|+\lambda)}
$$

Lemma 3.29. We have

$$
\int_{\mathbb{R}^{n}}\left|M^{d y}\left(f V^{+}\right)\right|^{2} d x \leqq C \cdot L \int_{\mathbb{R}^{n}}|f|^{2} V^{+} d x .
$$


Proof. Let $j$ be an integer. Suppose $Q_{j}^{k}$ 's are the maximal element among all dyadic cubes which satisfy

$$
\frac{1}{l(Q)^{n-1}} \int_{Q}|f| V^{+} d x>2^{j} \quad \text { and } \quad l(Q)<\inf _{x \in Q} \frac{C_{4}^{2} c_{0}}{m(x,|\mathbf{B}|+\lambda)}
$$

Then $Q_{j}^{k}$ are disjoint for a fixed $j$ and

$$
\left\{x \in \mathbb{R}^{n}: M^{d y}\left(f V^{+}\right)(x)>2^{J}\right\}=\bigcup_{k} Q_{j}^{k}
$$

It follows that

$$
\begin{aligned}
\int_{\mathbb{R}^{n}}\left|M^{d y}\left(f V^{+}\right)\right|^{2} d x & \leqq \sum_{j} 2^{2 j+2}\left|\left\{x \in \mathbb{R}^{n}: 2^{j}<M^{d y}\left(f V^{+}\right)(x) \leqq 2^{j+1}\right\}\right| \\
& =\sum_{j, k} 2^{2 j+2}\left|E_{j}^{k}\right|
\end{aligned}
$$

where

$$
E_{j}^{k}=Q_{j}^{k} \backslash\left\{x \in \mathbb{R}^{n}: M^{d y}\left(f V^{+}\right)(x)>2^{j+1}\right\}
$$

Thus,

$$
\begin{aligned}
& \int_{\mathbb{R}^{n}}\left|M^{d y}\left(f V^{+}\right)\right|^{2} d x \leqq 4 \sum_{j, k}\left\{\frac{1}{l\left(Q_{j}^{k}\right)^{n-1}} \int_{Q_{j}^{k}}|f| V^{+} d x\right\}^{2}\left|E_{j}^{k}\right| \\
& =C \sum_{j, k}\left\{\frac{1}{V^{+}\left(Q_{j}^{k}\right)} \int_{Q_{j}^{k}}|f| V^{+} d x\right\}^{2} \cdot V^{+}\left(E_{j}^{k}\right) \cdot \frac{V^{+}\left(Q_{j}^{k}\right)}{V^{+}\left(E_{j}^{k}\right)} \cdot \frac{\left|E_{j}^{k}\right|}{\left|Q_{j}^{k}\right|} \cdot \frac{V^{+}\left(Q_{j}^{k}\right)}{l\left(Q_{j}^{k}\right)^{n-2}} \\
& \leqq C \cdot L \sum_{j, k}\left\{\frac{1}{V^{+}\left(Q_{j}^{k}\right)} \int_{Q_{j}^{k}}|f| V^{+} d x\right\}^{2} \cdot V^{+}\left(E_{j}^{k}\right) \cdot \frac{V^{+}\left(Q_{j}^{k}\right)}{V^{+}\left(E_{j}^{k}\right)} \cdot \frac{\left|E_{j}^{k}\right|}{\left|Q_{j}^{k}\right|},
\end{aligned}
$$

where we have used the notation

$$
V^{+}(E)=\int_{E} V^{+}(x) d x .
$$

Now, note that, if $c_{0}$ is small,

$$
l\left(Q_{j}^{k}\right) \leqq \inf _{x \in Q_{j}^{k}} \frac{C_{4}^{2} c_{0}}{m(x,|\mathbf{B}|+\lambda)}<\inf _{x \in Q_{j}^{k}} \frac{\alpha}{m(x,|\mathbf{B}|)+\sqrt{\lambda}} .
$$

By (3.26),

$$
\frac{V^{+}\left(Q_{j}^{k}\right)}{V^{+}\left(E_{j}^{k}\right)} \cdot \frac{\left|E_{j}^{k}\right|}{\left|Q_{j}^{k}\right|} \leqq C
$$


It follows that

$$
\begin{aligned}
\int_{\mathbb{R}^{n}}\left|M^{d y}\left(f V^{+}\right)\right|^{2} d x & \leqq C \cdot L \sum_{j, k}\left\{\frac{1}{V^{+}\left(Q_{j}^{k}\right)} \int_{Q_{j}^{k}}|f| V^{+} d x\right\}^{2} \cdot V^{+}\left(E_{j}^{k}\right) \\
& \leqq C \cdot L \sum_{j, k} \int_{E_{j}^{k}}\left|M_{V^{+}}(f)\right|^{2} V^{+} d x \\
& \leqq C \cdot L \int_{\mathbb{R}^{n}}\left|M_{V^{+}}(f)\right|^{2} V^{+} d x
\end{aligned}
$$

where

$$
M_{V^{+}}(f)(x)=\sup _{\substack{Q \ni x \\ Q \text { dyadic }}} \frac{1}{V^{+}(Q)} \int_{Q}|f| V^{+} d y .
$$

The lemma then follows from the $L^{2}$ inequality for the dyadic Hardy-Littlewood maximal function in the space $L^{2}\left(\mathbb{R}^{n}, V^{+} d x\right)$. We remark that in the dyadic case, the doubling condition on the measure is not needed.

We now are in a position to give the

Proof of Theorem 3.1. We may assume that $\lambda>0$. By Lemma 3.4 and $M_{1} \leqq M_{p}$,

$$
\int_{\mathbb{R}^{n}}|V|\left|(H(\mathbf{a}, 0)+\lambda)^{-1 / 2} f\right|^{2} d x \leqq C \cdot M_{p} \int_{\mathbb{R}^{n}}|f|^{2} d x+C \int_{\mathbb{R}^{n}}|V|\left|T_{1}(|f|)\right|^{2} d x .
$$

By duality, the desired estimate of $T_{1}(|f|)$ follows from

$$
\int_{\mathbb{R}^{n}}\left|T\left(g V^{+}\right)\right|^{2} d x \leqq C \cdot M_{p} \int_{\mathbb{R}^{n}}|g|^{2} V^{+} d x .
$$

See (3.11). By Lemma 3.13, Lemma 3.21 and Lemma 3.29,

$$
\begin{aligned}
\int_{\mathbb{R}^{n}}\left|T\left(g V^{+}\right)\right|^{2} d x & \leqq C \int_{\mathbb{R}^{n}}\left|M\left(g V^{+}\right)\right|^{2} d x \leqq C \int_{\mathbb{R}^{n}}\left|M^{d y}\left(g V^{+}\right)\right|^{2} d x \\
& \leqq C \cdot L \int_{\mathbb{R}^{n}}|g|^{2} V^{+} d x .
\end{aligned}
$$

Finally, note that

$$
\begin{aligned}
L & =\sup _{Q} l^{2}(Q)\left(\frac{1}{|Q|} \int_{Q} V^{+}(x) d x\right) \\
& \leqq C \sup _{Q} l(Q)^{2}\left\{\sup _{Q^{\prime} \supseteq Q}\left(\frac{1}{\left|Q^{\prime}\right|} \int_{Q^{\prime}}|V|^{p} d x\right)^{1 / p}\right\} \\
& \leqq C \sup _{Q^{\prime}} l\left(Q^{\prime}\right)^{2}\left(\frac{1}{\left|Q^{\prime}\right|} \int_{Q^{\prime}}|V|^{p} d x\right)^{1 / p}=C \cdot M_{p},
\end{aligned}
$$

where $Q$ and $Q^{\prime}$ are dyadic cubes satisfying (3.9). The proof is complete. 


\section{The Proof of Theorem 0.11}

In this section we give the proof of Theorem 0.11 stated in the Introduction.

We begin with a lower bound for the operator $H(\mathbf{a}, 0)$.

Theorem 4.1. Under the same hypothesis as in Theorem 0.11 , we have, for $g \in$ $C_{0}^{1}\left(\mathbb{R}^{n}\right)$,

$$
\int_{\mathbb{R}^{n}}|m(x,|\mathbf{B}|) g(x)|^{2} d x \leqq C \sum_{j=1}^{n} \int_{\mathbb{R}^{n}}\left|\left(\frac{1}{i} \frac{\partial}{\partial x_{j}}-a_{j}\right) g(x)\right|^{2} d x .
$$

Theorem 4.1 follows from [Sh3, Theorem 2.7] by a rescaling argument similar to that in the proof of Theorem 2.6. We omit the details.

Definition 4.2. Let $\mathscr{B}$ be a collection of dyadic cubes in $\mathbb{R}^{n}$. For $Q, Q^{\prime} \in \mathscr{B}$, we say that $Q^{\prime}$ is a descendent of $Q$ if $Q^{\prime}$ is maximal with respect to the property of being properly contained in $Q$. We shall say $Q$ branches if $Q$ has at least two descendents.

The proof of the following lemma can be found in [F, pp. 156-157].

Lemma 4.3. Suppose $\mathscr{B}$ is a collection of dyadic cubes in $\mathbb{R}^{n}$. Let $\mathscr{B}_{0}$ be the subset of $\mathscr{B}$ consisting of (i) the maximal cubes in $\mathscr{B}$, (ii) the branching cubes in $\mathscr{B}$, (iii) the descendents of branching cubes in $\mathscr{B}$. Then the number of cubes in $\mathscr{B}_{0}$ is bounded by $C_{n} \cdot N$, where $N$ is the number of minimal cubes in $\mathscr{B}$.

We also need a lemma which may be found in [K-Sa, p. 224].

Lemma 4.4. Suppose $Q_{1}, \ldots, Q_{k}$ are pairwise disjoint dyadic subcubes of a dyadic cube $Q$ in $\mathbb{R}^{n}$. Then there are (not necessarily dyadic or disjoint) cubes $I_{1}, \ldots, I_{m}$ such that $Q \backslash \bigcup_{j=1}^{k} Q_{j}=\bigcup_{l=1}^{m} I_{l}$ and $m \leqq C_{n} \cdot k$.

For $\lambda \leqq 0$ and $\alpha>0$, let $\mathscr{A}=\mathscr{A}(|\mathbf{B}|, \alpha, \lambda)$ denote the collection of dyadic cubes $Q$ in $\mathbb{R}^{n}$ which satisfy

$$
l(Q)<\inf _{x \in Q} \frac{\alpha}{m(x,|\mathbf{B}|)+\sqrt{|\lambda|}} .
$$

Clearly, if $Q \in \mathscr{A}$, so does any dyadic subcube of $Q$.

We now give the

Proof of Theorem 0.11 Let $\mathscr{B}$ be the collection of all dyadic cubes $Q$ in $\mathscr{A}$ with the property

$$
l(Q)^{2}\left(\frac{1}{|Q|} \int_{Q}|V|^{p} d x\right)^{1 / p} \geqq c_{1}>0 .
$$

Suppose that $Q_{1}, Q_{2}, \ldots, Q_{\tilde{N}_{0}}$ are the minimal cubes in $\mathscr{B}$. We will show that there exists a subspace $\mathscr{H}$ of $L^{2}\left(\mathbb{R}^{n}\right)$ of codimension less than or equal to $C_{n} \tilde{N}_{0}$, such that, for any $g \in \mathscr{H}$,

$$
\sum_{j=1}^{n} \int_{\mathbb{R}^{n}}\left|\left(\frac{1}{i} \frac{\partial}{\partial x_{j}}-a_{j}\right) g\right|^{2} d x+|\lambda| \int_{\mathbb{R}^{n}}|g|^{2} d x \geqq \int_{\mathbb{R}^{n}}|V||g|^{2} d x .
$$


By the minimax principle, (4.6) implies that

$$
N(\lambda, H) \leqq C_{n} \tilde{N}_{0} \leqq C_{n} N_{0} \text { for } \lambda \leqq 0,
$$

where $N_{0}$ is the number of minimal (disjoint) dyadic cubes satisfying $(0.13)$ and (0.14) in Theorem 0.11. See Remark 0.18.

As in [F], we need to introduce some additional cubes $Q_{\tilde{N}_{0}+1}, Q_{\tilde{N}_{0}+2}, \ldots, Q_{M_{0}}$ to consist of (i) the maximal cubes in $\mathscr{B}$, (ii) the branching cubes in $\mathscr{B}$, (iii) the descendents of branching cubes in $\mathscr{B}$. It follows from Lemma 4.3 that $M_{0} \leqq C_{n} \tilde{N}_{0}$.

Now, let

$$
\begin{aligned}
& E_{0}=\mathbb{R}^{n} \backslash \bigcup_{j=1}^{M_{0}} Q_{j}, \\
& E_{j}=Q_{j} \backslash \bigcup_{\substack{k \neq j \\
Q_{k} \subset Q_{j}}} Q_{k}, \quad j=1,2, \ldots, M_{0} .
\end{aligned}
$$

Then $E_{j}$ are disjoint and $\mathbb{R}^{n}=\bigcup_{j=0}^{M_{0}} E_{j}$.

Let $V_{j}=V \chi_{E_{j}}$. The same argument as in [F, pp. 157-159] shows that

$$
l(Q)^{2}\left(\frac{1}{|Q|} \int_{Q}\left|V_{j}\right|^{p} d x\right)^{1 / p} \leqq C c_{1}
$$

for $0 \leqq j \leqq M_{0}$ and any cube $Q$ in $\mathscr{A}$. Furthermore, if $1 \leqq j \leqq M_{0}$, (4.8) holds for any dyadic cube in $\mathbb{R}^{n}$. Indeed, if $Q \subset Q_{j}$, then $Q \in \mathscr{A}$ since $Q_{j} \in \mathscr{A}$. If $Q_{j} \subset Q$, (4.8) follows from the fact that $\operatorname{supp} V_{j} \subset Q_{j}$. Here we have assumed that $p \leqq n / 2$, since Theorem 0.11 becomes stronger as $p$ decreases.

To prove (4.6), we first estimate the integral over $E_{0}$. By (4.8) and Theorem 3.1, we have

$$
\int_{E_{0}}|V||g|^{2} d x \leqq C c_{1}\left\{\sum_{j=1}^{n} \int_{\mathbb{R}^{n}}\left|\left(\frac{1}{i} \frac{\partial}{\partial x_{j}}-a_{j}\right) g\right|^{2} d x+|\lambda| \int_{\mathbb{R}^{n}}|g|^{2} d x\right\}
$$

for $g \in C_{0}^{1}\left(\mathbb{R}^{n}\right)$.

To deal with the case $1 \leqq j \leqq M_{0}$, we use Lemma 4.4 to obtain

$$
E_{j}=\bigcup_{k=1}^{m_{j}} I_{k}^{j}
$$

where $I_{k}^{j}$ are cubes (not necessarily dyadic or disjoint) of $\mathbb{R}^{n}$. Also the number of cubes in $\left\{I_{k}^{j}: 1 \leqq j \leqq M_{0}, 1 \leqq k \leqq m_{j}\right\}$ is bounded by $C_{n} \tilde{N}_{0}$.

Thus, as in [K-Sa], if $x \in I_{k}^{j}$ and

$$
\int_{I_{k}^{J}} f(y) d y=0
$$

we have

$$
|f(x)| \leqq C(-\Delta)^{-1 / 2}\left(|\nabla f| \chi_{I_{k}^{\prime}}\right)(x) \leqq C(-\Delta)^{-1 / 2}\left(|\nabla f| \chi_{E_{J}}\right)(x)
$$


Hence, since (4.8) holds for any dyadic cubes, we may use the trace inequality for $(-\Delta)^{-1 / 2}[\mathrm{~K}-\mathrm{Sa}$, Theorem 2.3] to obtain

$$
\int_{E_{J}}|V||f|^{2} d x \leqq C \int_{\mathbb{R}^{n}}\left|V_{j}\right|\left|(-\Delta)^{-1 / 2}\left(|\nabla f| \chi_{E_{j}}\right)\right|^{2} d x \leqq C c_{1} \int_{E_{J}}|\nabla f|^{2} d x
$$

Finally, for each $Q_{j}$, we construct $\mathbf{h}^{j}$ and $\Phi^{j}$ as in the proof of Theorem 0.26 , such that

$$
\mathbf{a}(x)=\mathbf{h}^{j}(x)+\nabla \Phi^{j}(x) \text { for } x \in Q_{j} .
$$

See (1.3-1.7).

We define

$$
\mathscr{H}=\left\{g \in L^{2}\left(\mathbb{R}^{n}\right): \int_{I_{k}^{\prime}} e^{-i \Phi^{\prime}} g(x) d x=0 \text { for } j=1,2, \ldots, M_{0}, 1 \leqq k \leqq m_{j}\right\}
$$

Then, $\mathscr{H}$ is a subspace of $L^{2}\left(\mathbb{R}^{n}\right)$ of codimension less than or equal to $C_{n} \tilde{N}_{0}$. If $g \in \mathscr{H} \cap \operatorname{Domain}(H)$, we have

$$
\begin{aligned}
\int_{E_{J}}|V||g|^{2} d x & =\int_{E_{j}}|V|\left|g e^{-i \Phi^{J}}\right|^{2} d x \leqq C c_{1} \int\left|\nabla\left(g e_{E_{J}}^{-i \Phi^{J}}\right)\right|^{2} d x \\
& \leqq C c_{1} \sum_{l=1}^{n} \int_{E_{J}}\left|\left(\frac{1}{i} \frac{\partial}{\partial x_{l}}-\frac{\partial \Phi^{j}}{\partial x_{l}}\right) g\right|^{2} d x \\
& \leqq C c_{1}\left\{\sum_{l=1}^{n} \int_{E_{J}}\left|\left(\frac{1}{i} \frac{\partial}{\partial x_{l}}-a_{l}\right) g\right|^{2} d x+\int_{E_{J}}\left|\mathbf{h}^{j}\right|^{2}|g|^{2} d x\right\} \\
& \leqq C c_{1}\left\{\sum_{l=1}^{n} \int_{E_{j}}\left|\left(\frac{1}{i} \frac{\partial}{\partial x_{l}}-a_{l}\right) g\right|^{2} d x+\int_{E_{J}}|m(x,|\mathbf{B}|) g|^{2} d x\right\},
\end{aligned}
$$

where we have used $\left|\mathbf{h}^{j}(x)\right| \leqq C m(x,|\mathbf{B}|)$ on $Q_{J}$ in the last inequality. This, together with (4.9), yields that

$$
\begin{aligned}
& \int_{\mathbb{R}^{n}}|V||g|^{2} d x \\
& \quad \leqq C c_{1}\left\{\sum_{l=1}^{n} \int_{\mathbb{R}^{n}}\left|\left(\frac{1}{i} \frac{\partial}{\partial x_{l}}-a_{l}\right) g\right|^{2} d x+\int_{\mathbb{R}^{n}}|m(x,|\mathbf{B}|) g|^{2} d x+|\lambda| \int_{\mathbb{R}^{n}}|g|^{2} d x\right\} .
\end{aligned}
$$

The desired estimate (4.6) then follows from Theorem 4.1 by choosing $c_{1}$ sufficiently small.

The proof of Theorem 0.11 is complete. 


\section{References}

[A-H-S] Avron, J, Herbst, I, Simon, B.: Schrödinger Operators with Magnetic Fields I General Interaction Duke Math J 45(4), 847-883 (1978)

[C-W] Chanillo, S, Wheeden, R.: $L^{p}$ Estimates for Fractional Integrals and Sobolev Inequalities with Applications to Schrödinger Operators Comm in P D E 10, 1077-1116 (1985)

[F] Fefferman, C: The Uncertainty Principle Bull Am Math Soc 9, 129-206 (1983)

[H] Helffer, B : Semi-Classical Analysis for the Schrödinger Operator and Applications Lecture Notes in Math 1336, Berlin-Heidelberg-New York: Springer Verlag, 1988

[H-N] Helfer, B , Nourrigat, J : Hypoellipticité maximale pour des opérateurs polynômes de champs de vecteurs Progress in Math 58, Boston: Birkhäuser, 1985

[I] Iwatsuka, A : Magnetic Schrödinger Operators with Compact Resolvent J Math Kyoto Univ. 26(3), 357-374 (1986)

[K-Sa] Kerman, R, Sawyer, E : The Trace Inequality and Eigenvalue Estimates for Schrödinger Operators Ann Inst Fourier(Grenoble) 36(4), 207-228 (1986)

[L-S] Leinfelder, H, Simader, C : Schrödinger Operators with Singular Magnetic Vector Potentials Math Z 176, 1-19 (1981)

[M-R] Mohamed, A, Raikov, G: On the Spectral Theory of the Schrödinger Operator with Electronmagnetic Potential. In: Pseudo-Differential Calculus and Mathematical Physics, edited by M Demuth, E Schrohe and B W Schulze, Berlin: Akademie Verlag, 1994, pp 298-390

[R] Robert, D : Comportement asymptotique des valurs propres d'opérateurs du type Schrödinger á potentiel "dégénéré" J Math Pures Appl 61(9), 275-300 (1982)

[Sh1] Shen, Z: $L^{p}$ Estimates for Schrödinger Operators with Certain Potentials. Ann Inst Fourier (Grenoble) 45(2), 513-546 (1995)

[Sh2] Shen, Z: On the Eigenvalue Asymptotics of Schrödinger Operators Preprint (1995)

[Sh3] Shen, Z: Eigenvalue Asymptotics and Exponential Decay of Eigenfunctions for Schrödinger Operators with Magnetic Fields Trans Am Math Soc (to appear)

[Sh4] Shen, Z: Estimates in $L^{p}$ for Magnetic Schrödinger Operators. Preprint (1995)

[Si1] Simon, B : Schrödinger Semigroups Bull Am Math Soc. 7(3), 447-525 (1982)

[Si2] Simon, B : Functional Integration and Quantum Physics New York: Academic Press, 1979

[Si3] Simon, B : Nonclassical Eigenvalue Asymptotics J Funct Anal 53, 84-98 (1983)

[St1] Stein, E : Singular Integrals and Diffentiability Properties of Functions Princeton, NJ: Princeton Univ Press, 1970

[St2] Stein, E: Harmonic Analysis: Real-Variable Method, Orthogonality, and Oscillatory Integrals Princeton, NJ: Princeton Univ Press, 1993 